Check for updates

Cite this: Chem. Sci., 2019, 10, 4914

๑ All publication charges for this article have been paid for by the Royal Society of Chemistry

\section{A H-bond strategy to develop acid-resistant photoswitchable rhodamine spirolactams for super-resolution single-molecule localization microscopy $\dagger$}

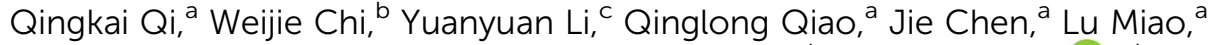 \\ Yi Zhang, ${ }^{a}$ Jin Li, ${ }^{a}$ Wei Ji, ${ }^{c}$ Tao Xu, ${ }^{* c}$ Xiaogang Liu, ${ }^{* b}$ Juyoung Yoon (iD *d \\ and Zhaochao Xu iD *a
}

\begin{abstract}
Rhodamine spirolactam based photoswitches have been extensively applied in super-resolution singlemolecule localization microscopy (SMLM). However, the ring-opening reactions of spirolactams are cross-sensitive to acid, limiting their photoswitch use to neutral $\mathrm{pH}$ conditions. In addition, the ringclosing reactions of spirolactams are environment-sensitive and slow (up to hours), virtually making rhodamine spirolactams caged fluorescent dyes instead of reversible photoswitches in SMLM. Herein, by introducing hydrogen bonds to stabilize spirolactams, we report a series of acid-resistant rhodamine spirolactams with accelerated ring-closing reactions from fluorescent xanthyliums to non-fluorescent spirolactams, endowing them with good photoswitchable properties even in acidic environments. By further substitution of 6-phenylethynyl naphthalimide on the spirolactam, we shifted the photoactivation wavelength into the visible region (>400 nm). Subsequently, we have successfully applied these dyes in labeling and imaging the cell surface of Bacillus subtilis at $\mathrm{pH} 4.5$ using SMLM.
\end{abstract}

Received 15th March 2019

Accepted 4th April 2019

DOI: $10.1039 / c 9 s c 01284 b$

rsc.li/chemical-science

\section{Introduction}

Super-resolution fluorescence microscopy breaks the diffraction limit and achieves nanometre-scale resolution. ${ }^{1}$ The fundamental principle underlying this technique relies on the "dark" and "fluorescent" pair states of fluorescent markers, enabling temporal separation of adjacent molecules in a subdiffraction-sized region. The requirements for both dark/ emitting state-switching and outstanding emission properties (including high brightness and photostability), particularly for super-resolution single-molecule localization microscopy (SMLM), ${ }^{2}$ have driven the creation of improved photoswitchable

${ }^{a}$ CAS Key Laboratory of Separation Science for Analytical Chemistry, Dalian Institute of Chemical Physics, Chinese Academy of Sciences, Dalian 116023, China.E-mail: zcxu@ dicp.ac.cn

${ }^{b}$ Singapore University of Technology and Design, 8 Somapah Road, Singapore 487372, Singapore. E-mail: xiaogang_liu@sutd.edu.sg

${ }^{c}$ National Laboratory of Biomacromolecules, Institute of Biophysics, Chinese Academy of Sciences, Beijing 100101, China. E-mail: xutao@ibp.ac.cn

${ }^{d}$ Department of Chemistry and Nano Science, Ewha Womans University, Seoul 120750, Korea.E-mail:jyoon@ewha.ac.kr

$\dagger$ Electronic supplementary information (ESI) available: Data-mining of 3D molecular structures of rhodamine spiroamides, computational results, general information on synthesis and characterization, Fig. S1-S26 and Tables S1-S7 (PDF), and Movies 1 and 2 showing the 3D-SMLM imaging of Bacillus subtilis cells. CCDC 1582847, 1901370-1901372. For ESI and crystallographic data in CIF or other electronic format see DOI: 10.1039/c9sc01284b fluorescent probes via two means: combining a photochromic moiety with excellent fluorophores ${ }^{3}$ and incorporating fluorescent fragments in photochromic compounds. ${ }^{4}$

Among various photoswitchable fluorophores, rhodamine spirolactams have attracted considerable research interest, owing to their excellent photophysical properties and biocompatibility. These dyes were widely applied in constructing various chemosensors for $\mathrm{pH}$, metal ions and bioactive molecules, based on ring-opening reactions from colorless and nonfluorescent spirolactams to colored and strongly fluorescent xanthyliums. ${ }^{5}$ Since their photoswitching properties were first reported in 1977, rhodamine spirolactams have been used as photochromic materials. ${ }^{6}$ Recently, owing to dark/emitting state switching, rhodamine spirolactams have also been successfully deployed in SMLM. ${ }^{7}$ While many exciting superresolution imaging applications have been demonstrated, ${ }^{\mathbf{8}}$ two significant challenges limit the application of rhodamine spirolactams. First, rhodamine spirolactams are strictly limited to neutral $\mathrm{pH}$ environments, due to their considerable acidactivated switching to fluorescent xanthyliums (the open form) across a wide acidic $\mathrm{pH}$ range (usually $2.0<\mathrm{pH}<6.5$ ) (Fig. 1a). ${ }^{5}$ As these dyes enter an acidic environment (such as in lysosomes ( $\mathrm{pH}$ 4.5-6.0), endosomes ( $\mathrm{pH}$ 5.0-6.5), or locations next to proton-donating groups in proteins), acid-activation becomes substantial, leading to the disablement of photoactivation. Yet, low $\mathrm{pH}$ or acidic environments are ubiquitous in 
biological systems. Intracellular acidification is associated with many critical diseases (such as cancer). ${ }^{9}$ Second, environmental changes greatly alter the lifetime of the open form, which ranges from milliseconds to hours. ${ }^{6,7 a, c}$ With a long "open form" lifetime in aqueous solutions, photobleaching reactions could considerably compete with thermal fading ring-closing reactions, compromising the photostability of rhodamine spirolactams. ${ }^{6 \boldsymbol{c}, 10}$ Moreover, on the timescale of seconds or longer, the photoswitching reversibility of rhodamine spirolactams is virtually negligible for image acquisition purposes; these dyes effectively behave as typical caged fluorescent compounds: they are activated, imaged, and then bleached (Fig. 1a). ${ }^{7 a}$ By introducing a lag-time in between frames to allow relaxation, these dyes could be used as localized markers several times. In a landmark paper, a spontaneously blinking fluorophore based on an intramolecular spirocyclization reaction was reported to increase reversible photoswitching cycles and allow long-term dynamic studies in live cells. ${ }^{10}$ In a recent study, the photoswitch of rhodamine spirolactam was combined with fluxionality to enable long time-lapse SMLM imaging in live cells. ${ }^{i}$ However, these fluorophores were still acid sensitive or $\mathrm{pH}$ dependent, limiting their application to neutral $\mathrm{pH}$ environments. Consequently, dedicated photoswitchable rhodamine spirolactams with acid immunity and short "open form" lifetime are required for SMLM.

Herein, we report a series of acid-resistant rhodamine spirolactams with a dedicated photoswitchable property and short "open form" lifetime. These characteristics are attributed to the substitution of 3-amino groups in the carboxyphenyl ring and associated intramolecular hydrogen bonding in spirolactams (Fig. 1b). Inspired by Moerner's work, ${ }^{7 c}$ we further extended the photoactivation wavelength of our dyes into the visible region (>400 nm), via conjugation with 6-phenylethynyl naphthalimide. The excellent acid-resistance and visible-light photoswitching make our rhodamine spirolactams suitable for SMLM imaging, and more importantly, generate reliable fluorescence signals even in acidic environments.

\section{Results and discussion}

\section{Molecular design strategy}

Our strategy of introducing intramolecular hydrogen bonding to increase the acid-resistance of spirolactams and shorten the "open form" lifetime originated from three facts: (1) the acidactivated ring-opening reaction is initiated with the protonation of the carbonyl oxygen (Fig. 1a); ${ }^{1}$ (2) the resulting ring opening reaction is associated with a rotation of the amide group (-CONR) with respect to the phenyl ring (Fig. 1a, S1; Tables S1 and S2 $\dagger$ ); (3) introducing an electron-donating group into spirolactams reduces the driving force of ring-opening reactions. ${ }^{11}$ We anticipated that the intramolecular hydrogen bonding would reduce the reactivity of carbonyl oxygen with exterior acid and lock the spirolactam group. In addition, both the suppressed rotation of the amide group and the incorporation of an electron-donating group contributed to the stabilization of the closed form. Accordingly, we synthesized a

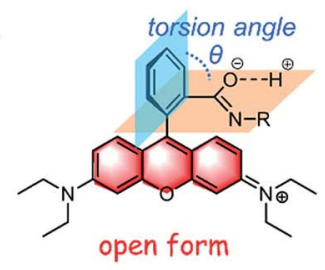

b
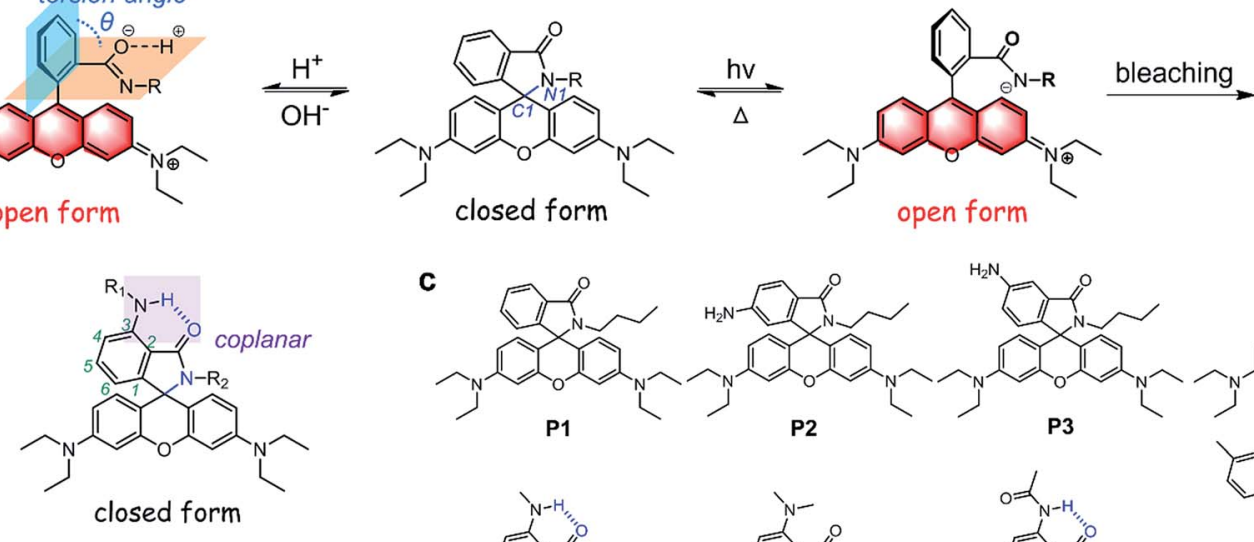

$\mathrm{H}_{2} \mathrm{~N}$

,


compounds P1-P6 to verify our hypothesis (Fig. 1c and Schemes S1-S3†). Compounds P4 and P5 possessed 3-substituted NHR groups which were expected to form intramolecular hydrogen bonds with the lactam moiety, while the rest served as control compounds without intramolecular hydrogen bonds.

\section{Characterization of the acid-resistance of rhodamine spirolactams}

We first examined the acid resistance of P1-P6. With the addition of $\mathrm{CF}_{3} \mathrm{COOH}$, a new emission band centered at $560 \mathrm{~nm}$ in the absorption spectra of P1-P3 and P6 was observed (Fig. 2a, b, S2 and S3 $\dagger$ ). Accordingly, the solutions of P1-P3 and P6 displayed noticeable color changes from colorless to pink, indicating the acid-controlled xanthylium formation. In stark contrast, the UV-vis absorption spectra of $\mathbf{P 4}$ and $\mathbf{P 5}$ did not show obvious changes in the visible region, and the solutions almost remained colorless (Fig. 2a, b and $\mathrm{S} 2 \dagger$ ). These results indicated that the fluorescence switching of P4 and P5 was far less acid-sensitive, while that of P1-P3 and P6 was acidsensitive.

\section{Verification of the $\mathbf{H}$-bond mechanism}

We next conducted NMR and crystallographic experiments to examine the existence of intramolecular hydrogen bonding (Fig. 2c, d and Table S3 $\dagger$ ). ${ }^{1} \mathrm{H}$ NMR spectra show large downfield shifts of the resonance of NH protons in P4 (5.30) and P5 (6.75), compared with those in P2 (3.83) and P3 (3.83) (Fig. 2c). The crystal structure of $\mathbf{P 4}$ directly revealed strong intramolecular hydrogen bonding between $\mathrm{NH}$ and carbonyl oxygen (distance: $2.347 \AA$ A angle: $128.61^{\circ}$ ) (Fig. $2 d$ ), which explained the downfield shift of NH in P4. Though we failed to obtain the crystal structure of P5, we believed that the much larger downfield shift of $\mathrm{NH}$ in $\mathbf{P 5}$ indicated even stronger intramolecular hydrogen bonding in P5. According to the absorption and emission spectral evolution of $\mathbf{P 4}$ and $\mathbf{P 5}$ (after the addition of $\mathrm{CF}_{3} \mathrm{COOH}$ ), P5 was more acid-resistant than $\mathbf{P 4}$, which should be ascribed to the stronger intramolecular hydrogen bonding (Fig. S2-S4†). In addition, we also noticed weak intramolecular $\mathrm{CH} \cdots \mathrm{O}$ interactions in P6 (distance: $2.249 \AA$ A; angle: $130.80^{\circ}$ ). The above results clearly demonstrate that intramolecular hydrogen bonding plays a key role in enhancing the acid-resistance of rhodamine spirolactams.

We also performed density functional theory (DFT) calculations on P1-P6 to rationalize the hydrogen-bond induced acidresistance. As expected, optimized molecular geometries show that intramolecular hydrogen bonds are present only in $\mathbf{P 4}$ and P5 (Fig. S5†). Moreover, by modelling eight representative protonation configurations for each compound to fully simulate their responses in an acidic aqueous environment (Fig. S6 $\dagger$ ), our results show that among the control compounds, fluorescent open-ring structures possess significantly lower Gibbs free energy than non-emissive closed-ring structures by up to $0.19 \mathrm{eV}$ (Table S4 and Fig. S7-S12 $\dagger$ ). Therefore, DFT calculations suggest that the emissions of these control compounds could easily turn on in an acidic environment. In contrast, the closedring structures of P4 and P5 are relatively more stable than
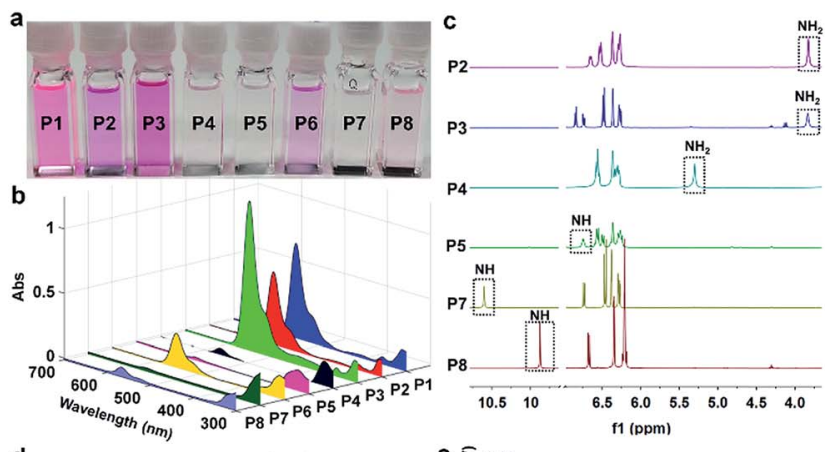
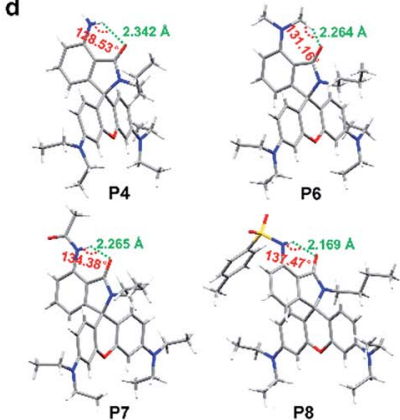

g

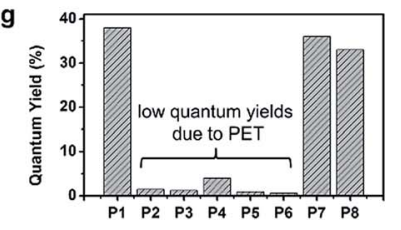

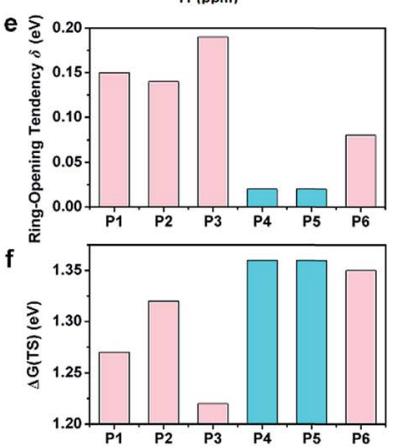

h

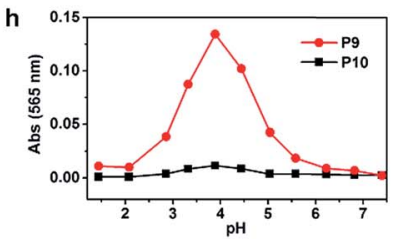

Fig. 2 (a) Photograph and (b) UV-vis absorption spectra of P1-P8 in $\mathrm{CH}_{2} \mathrm{Cl}_{2} / \mathrm{CH}_{3} \mathrm{OH}(9 / 1, \mathrm{v} / \mathrm{v})$, two hours after the addition of $2.3 \mu \mathrm{L}$ of $\mathrm{CF}_{3} \mathrm{COOH}$ (1000 equiv.). (c) Local ${ }^{1} \mathrm{H}$ NMR spectra of P2-P5 and P7P8. (d) Single-crystal structures and intramolecular hydrogen bonds of $\mathrm{P} 4, \mathrm{P} 7$ and $\mathrm{P} 8$ and weak $\mathrm{CH} \cdots \mathrm{O}$ interactions in P6. (e) Calculated ringopening tendencies of P1-P6 in acidic solution. (f) Calculated energy barriers during the ring-opening process of neutral P1-P6. (g) Fluorescence quantum yields of $\mathrm{P} 1-\mathrm{P} 8$ in $\mathrm{CH}_{2} \mathrm{Cl}_{2} / \mathrm{CH}_{3} \mathrm{OH}(9 / 1, \mathrm{v} / \mathrm{v})$ solution. (h) The absorption intensities at $565 \mathrm{~nm}$ of P9 and P10 as a function of $\mathrm{pH}$ in water.

those of P1-P3 and P6 by $\sim 0.12 \mathrm{eV}$, suggesting that these structures are resistant to acid-activation. We further calculated the difference in Gibbs free energy between the most stable closed-ring and open-ring protonated structures $(\delta)$ to quantify the tendency of ring-opening in P1-P6. Herein, a large $\delta$ corresponds to a strong thermodynamic driving force for ringopening reactions to proceed. Indeed, our results showed that $\mathbf{P 4}$ and P5 exhibited a significantly lower ring-opening tendency than P1-P3 and P6 did (Fig. 2e). We also computed the energy barriers during the ring-opening reactions of P1-P6 (Table S5 and Fig. S13-S19†). Our results show that the barriers in $\mathbf{P 4}$ and P5 are higher than those of the control compounds, due to the presence of intramolecular hydrogen bonds (Fig. 2f). All these computational results rationalize that $\mathbf{P 4}$ and $\mathbf{P 5}$ possess significantly enhanced acid-resistance towards fluorescence activation. At the same time, we expected that $\mathbf{P 4}$ and $\mathbf{P 5}$ remained photoswitchable, given the high energy of photoactivation light. 
Improving quantum yields and water solubility of rhodamine spirolactams

Unfortunately, the quantum yields of P2-P6 are low (Fig. 2g), possibly quenched by photo-induced electron transfer (PET) from the 3-aminophenyl moiety to the xanthene scaffold. To enhance brightness and preserve acid-resistance, we synthesized P7 and P8 (Scheme S4 $\dagger$ ) with 3-acetamido and 3-toluenesulfonamide substituents to inhibit PET, respectively. As a result, the quantum yields of $\mathbf{P 7}$ and $\mathbf{P 8}$ were significantly improved (Fig. 2g). At the same time, $\mathbf{P 7}$ and $\mathbf{P 8}$ remained acidresistant (Fig. 2a and b). ${ }^{1} \mathrm{H}$ NMR analysis and the single crystal structures of $\mathbf{P 7}$ and $\mathbf{P 8}$ clearly showed the existence of intramolecular hydrogen bonds (Fig. 2c and d). Notably, the stronger intramolecular hydrogen bonding in P7 than that in $\mathbf{P 8}$ resulted in stronger acid-resistance of $\mathbf{P 7}$ than that of $\mathbf{P 8}$ (Fig. S2-S4†). Owing to its high brightness and excellent acid-resistance, we chose $\mathbf{P 7}$ for further derivations and applications.

Subsequently, we synthesized water-soluble compounds P9 and P10 with polyethylene glycol (PEG) groups to verify the applicability of our strategy in water (Scheme S5 $\dagger$ ). As shown in Fig. $2 \mathrm{~h}$ and $\mathrm{S} 20, \uparrow$ the UV-vis absorption and emission intensities of the open form of P9 substantially increased when the $\mathrm{pH}$ was decreased from 7.40 to 3.89 , and then decreased when the $\mathrm{pH}$ was decreased from 3.89 to 1.44. In contrast, P10 did not show any noticeable absorption and fluorescence bands in the visible region in the $\mathrm{pH}$ range from 1.44 to 7.40 (Fig. $2 \mathrm{~h}$ and $\mathrm{S} 20 \dagger$ ). These results indicated that our strategy to increase the acid-resistance of rhodamine spirolactams worked well in aqueous solutions and was suitable for biological systems.

\section{Evaluation of open form lifetime}

We next evaluated the lifetime of the open forms via laser flash photolysis (Table S6 and Fig. S21†). It's reported that the lifetime of the open form was greatly affected both by the environment and molecular structures., ${ }^{6,7 a, c}$ The open form has a lifetime of a few milliseconds in polar solvents. ${ }^{6}$ In polyvinylalcohol, the lifetime of the open form was reported to lengthen to hours. ${ }^{7 a}$ By introducing aromatic substituents on the lactam nitrogen, the open isomer with the charge at the amide nitrogen atom can be stabilized and then it has a longer lifetime. ${ }^{6 \boldsymbol{b}, 7 \boldsymbol{a}, \boldsymbol{c}}$ In our experiments, the lifetime of P1 vs. P7 in $\mathrm{CH}_{2} \mathrm{Cl}_{2}$ was too short to be examined (Table S6 $\dagger$ ). But in dioxane, the lifetime was detected to be in the range of $60 \mathrm{~ns}$. Importantly, we found that the intramolecular hydrogen bonding could shorten the lifetime of the open forms (62.5 ns for P1 and $59.7 \mathrm{~ns}$ for P7). The effect of intramolecular hydrogen bonding on lifetime becomes more significant when an aromatic substituent is introduced on the lactam nitrogen. Compared with the $200 \mathrm{~ms}$ lifetime of $\mathrm{N}$-phenylrhodaminelactame in dioxane, ${ }^{\boldsymbol{a}, \boldsymbol{b} \boldsymbol{b}}$ the lifetime of the reference compound P11 was much shorter, measured to be $\sim 31.5 \mathrm{~ns}$ in the same solvent. These results indicated that the thermal fading processes in our dyes may compete with photobleaching.

\section{In vitro bioimaging experiments}

With this progress, we continued to synthesize P12 and P13 to validate our design strategy with in vitro bioimaging experiments (Fig. 3 and Scheme S7†). The "control" probe $\mathbf{P 1 2}$ and the "experimental" probe $\mathbf{P 1 3}$ are coupled with a morpholine moiety, which enables these probes to become protonated and accumulated in lysosomes. The $\mathrm{pH}$ value of lysosomes is in the range of 4.0-6.0, which provides a good platform to test the acid-resistance of rhodamine spirolactams. After incubation for $30 \mathrm{~min}, \mathbf{P 1 2}$ emitted strong fluorescence in MCF-7 cells (Fig. S22 $\dagger$ ). Subsequently, we performed co-localization experiments of P12 (the red channel) with LysoTracker Green DND-26 (LTG; the green channel), a commercially available lysosome probe (Fig. 3a). The good overlap of fluorescence signals in the red and green channels demonstrates that P12 was distributed in the lysosomes of MCF-7 cells. Overall, these results show that the acidic environment in lysosomes has activated intensive fluorescence in the control probe $\mathbf{P 1 2}$.

In contrast, after incubating MCF-7 cells with P13 for 2 hours, we did not observe any fluorescence signals (Fig. S22 $\dagger$ ). To ensure the presence of P13 in lysosomes, continuous UV

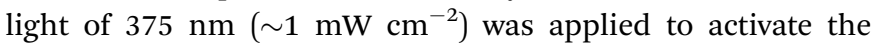
fluorescence of P13. By increasing the illumination time, we noticed a gradual increase of fluorescence signals in the red channel (Fig. 3b). These signals overlapped with the fluorescence of the lysosome probe LTG in the green channel. It is thus

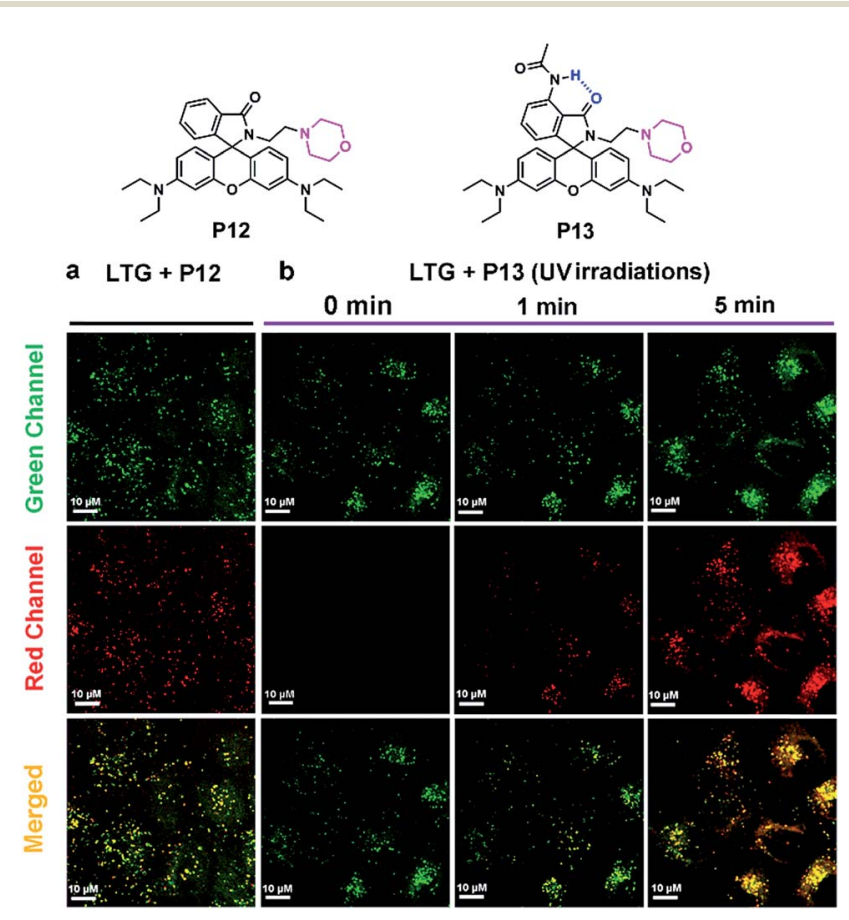

Fig. 3 Confocal images of MCF-7 cells stained with LTG $(0.1 \mu M)$ and (a) P12 $(10 \mu \mathrm{M})$ or (b) P13 $(10 \mu \mathrm{M})$; green channel: excitation at $488 \mathrm{~nm}$, emission collected from 500 to $550 \mathrm{~nm}$; red channel: excitation at $561 \mathrm{~nm}$, emission collected from 580 to $653 \mathrm{~nm}$. Insets: chemical structures of P12 and P13. The Pearson coefficients are high, 0.905 (LTG with P12) and 0.925 (LTG with P13 activated by UV light for 5 min), respectively. 
clear that P13 maintains acid-resistance in lysosomes and possesses a dedicated photoactivation property for cellular imaging. It's worth mentioning that the fluorescent form of P13 in lysosomes did not thermally revert back to the closed form in a short time. Considering the environmental sensitivity of the open form, this is probably because a large number of P13 accumulated in lysosomes, and many xanthyliums formed under continuous strong UV irradiation tended to stabilize each other, thus slowing down the ring-close reactions. The photoactivation experiments of $\mathbf{P 1}$ and $\mathbf{P} 7$ in $\mathrm{CH}_{2} \mathrm{Cl}_{2}$ may support our hypothesis. The open form lifetimes of $\mathbf{P 1}$ and $\mathbf{P 7}$ in $\mathrm{CH}_{2} \mathrm{Cl}_{2}$ were too short to observe a large amount of open isomers. But if we excited these compounds in $\mathrm{CH}_{2} \mathrm{Cl}_{2}$ solution with continuous UV irradiation, simultaneously photoactivated xanthyliums may accumulate and then significantly slow down the decay dynamics of $\mathbf{P 1}$ and P7, where the lifetime of the open isomers was on the order of min (Fig. S23†).

\section{Photoactivation of rhodamine spirolactams using visible light}

Encouraged by the success of P13 in bioimaging applications, we moved on to extend the photoactivation wavelength of rhodamine spirolactams into the visible region for cell imaging. We thus synthesized P14-P17 by conjugating a 6-phenylethynyl naphthalimide moiety to the spirolactam (Fig. 4a and Scheme S8 $\dagger$ ). The enlarged $\pi$-conjugation extends the UV-vis absorption spectra of P14-P17 into the visible region, with a peak absorption wavelength at $\sim 400 \mathrm{~nm}$ (Fig. $4 \mathrm{~b}$ ). It is thus possible to photoactivate these dyes with a $405 \mathrm{~nm}$ laser and read out the

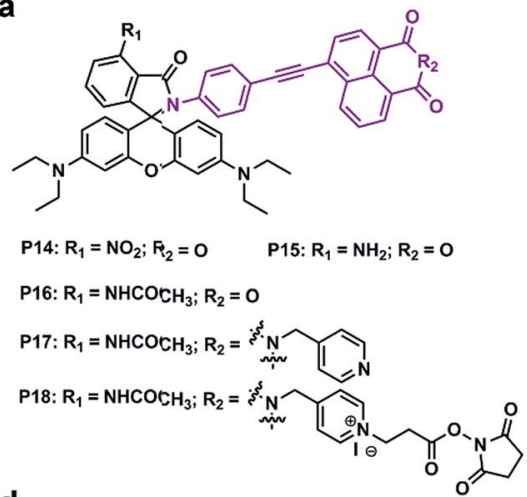

d
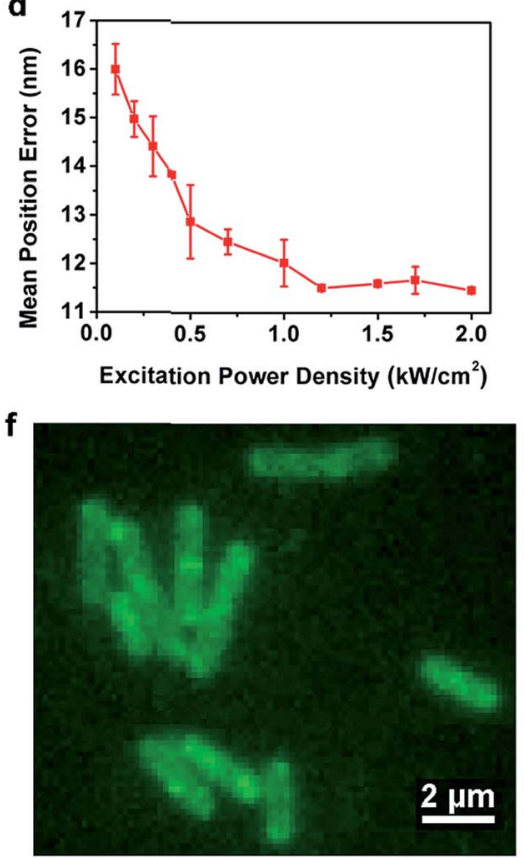

b
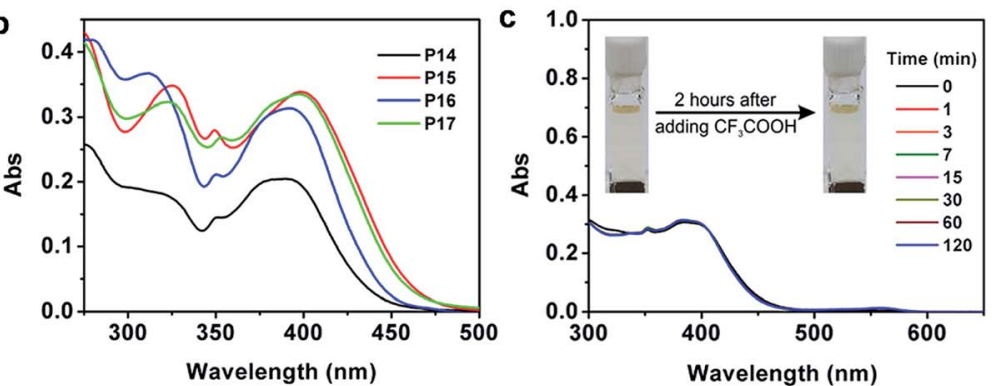

e
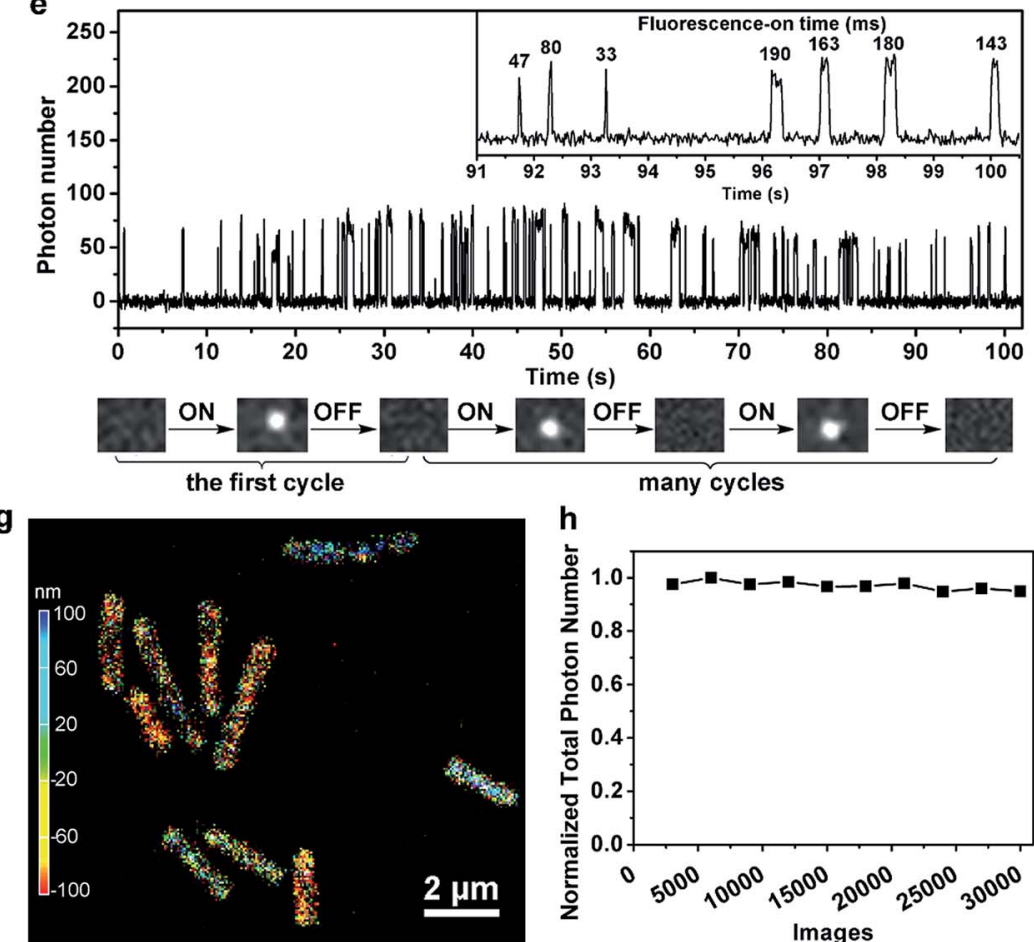

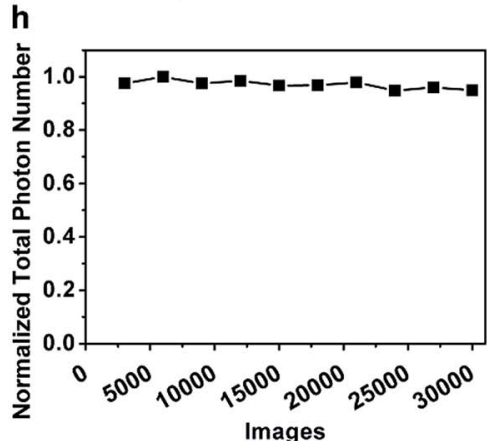

Fig. 4 (a) Chemical structures of P14-P18. (b) UV-vis absorption spectra of P14-P17 $\left(10^{-5} \mathrm{M}\right)$ in DMSO. (c) Time-dependent UV-vis absorption spectra of $\mathrm{P} 17\left(10^{-5} \mathrm{M}\right)$ in $\mathrm{CH}_{2} \mathrm{Cl}_{2} / \mathrm{CH}_{3} \mathrm{OH}(9 / 1, \mathrm{v} / \mathrm{v})$ before and after the addition of $\mathrm{CF}_{3} \mathrm{COOH}(2.3 \mu \mathrm{L}$, 1000 equiv.); the insets show the photographs of $\mathrm{P} 17$ before and two hours after adding $\mathrm{CF}_{3} \mathrm{COOH}$. (d) Single molecule localization precision of P17 at various excitation laser power densities. (e) Single-molecule fluorescence time traces of P18. (f) Conventional diffraction-limited fluorescence image of live Bacillus subtilis cells stained with P18 in pH 4.5 buffer solution showing average fluorescence over 30000 frames (20 ms integration time per frame). (g) 3D-SMLM image of live Bacillus subtilis cells stained with $\mathrm{P} 18$ in $\mathrm{pH} 4.5$ buffer solution; the SMLM image contains ca. $5.7 \times 10^{5}$ localized positions, each plotted as a Gaussian with the full width at half-maximum (FHWM) in the $X$ and $Y$ direction. (h) Normalized total photon output in a set of 30000 -frame images. 
fluorescence signals with an excitation laser of $561 \mathrm{~nm}$. The visible photoactivation wavelength is critical for minimizing phototoxicity in live-cell imaging.

Among P14-P17, we selected P17 for further experiments, foreseeing its excellent acid-resistance and good quantum yield. Indeed, P17 remained colorless in acidic medium (Fig. 4c). We then doped P17 into a PMMA film at a low concentration to characterize its single-molecule fluorescence properties. We applied a continuous $405 \mathrm{~nm}$ laser light $\left(60 \mathrm{~W} \mathrm{~cm}^{-2}\right)$ to photoactivate P17 (Fig. S24†), and a $561 \mathrm{~nm}$ laser light to generate fluorescence from activated P17. Our experiments showed that a laser excitation power of $\sim 1.2 \mathrm{~kW} \mathrm{~cm}$ c $^{-2}$ at $561 \mathrm{~nm}$ led to a localization precision of the single molecule of $\sim 12 \mathrm{~nm}$ in PMMA (Fig. 4d and S25 $\dagger$ ). Subsequent experiments showed that good imaging quality could be achieved by reducing the photoactivation light intensity at $405 \mathrm{~nm}$ down to $1.8 \mathrm{~W} \mathrm{~cm}^{-2}$ in a cellular environment.

\section{Super-resolution imaging of Bacillus subtilis in acidic environments}

To fully demonstrate the imaging applications of our photoswitchable dyes, we linked $N$-hydroxysuccinimide ester to P17, resulting in the formation of P18 (Fig. 4a and Scheme S9†). Laser flash photolysis showed that the open form lifetime of P18 was $105 \mathrm{~ns}$ in aqueous solutions with 5\% DMSO (Table S6 and
Fig. S21 $\dagger$ ). In a single molecule fluorescence imaging experiment with a $561 \mathrm{~nm}$ laser $\left(0.36 \mathrm{~kW} \mathrm{~cm}^{-2}\right)$ as an excitation source, P18 labeled on a glass coverslip showed reversible fluorescence blinking 100 times (Fig. 4e). Moreover, in this experiment the fluorescence-on time of P18 increased to $\sim 100$ ms-scale, probably owing to the environmental sensitivity of the open form lifetime. For example, this lifetime of a rhodamine spirolactam increased from milliseconds in polar solvents to hours in polyvinyl alcohol. ${ }^{7 a}$ The reversible blinking and good fluorescence signals inspired us to label P18 on the membrane surface of Gram-positive Bacillus subtilis cells in PBS solution ( $\mathrm{pH}$ 4.5), since these bacteria can maintain satisfactory activity in an acidic $\mathrm{pH}$ environment. We expected that P18 could specifically label bacterial membranes for the following reasons: (1) the positively charged $\mathbf{P 1 8}$ could bind with the anionic bacterial membranes through electrostatic interactions, and these interactions could further inhibit the diffusion of P18 through cell membranes; (2) the NHS ester of P18 could rapidly label the amino groups in the membrane proteins; and (3) the hydrophobic 1,8-naphthalimide group may intercalate into the membrane bilayer. We then imaged acid-resistant Bacillus subtilis cells, using a continuous $405 \mathrm{~nm}$ laser $(1.8 \mathrm{~W}$ $\left.\mathrm{cm}^{-2}\right)$ as photoactivation light and a $561 \mathrm{~nm}$ laser $\left(1.2 \mathrm{~kW} \mathrm{~cm}^{-2}\right)$ as an excitation source. The fluorescence of P18 exhibited an excellent photo-blinking property, allowing the reconstruction of the super-resolution image via single-molecule localization.
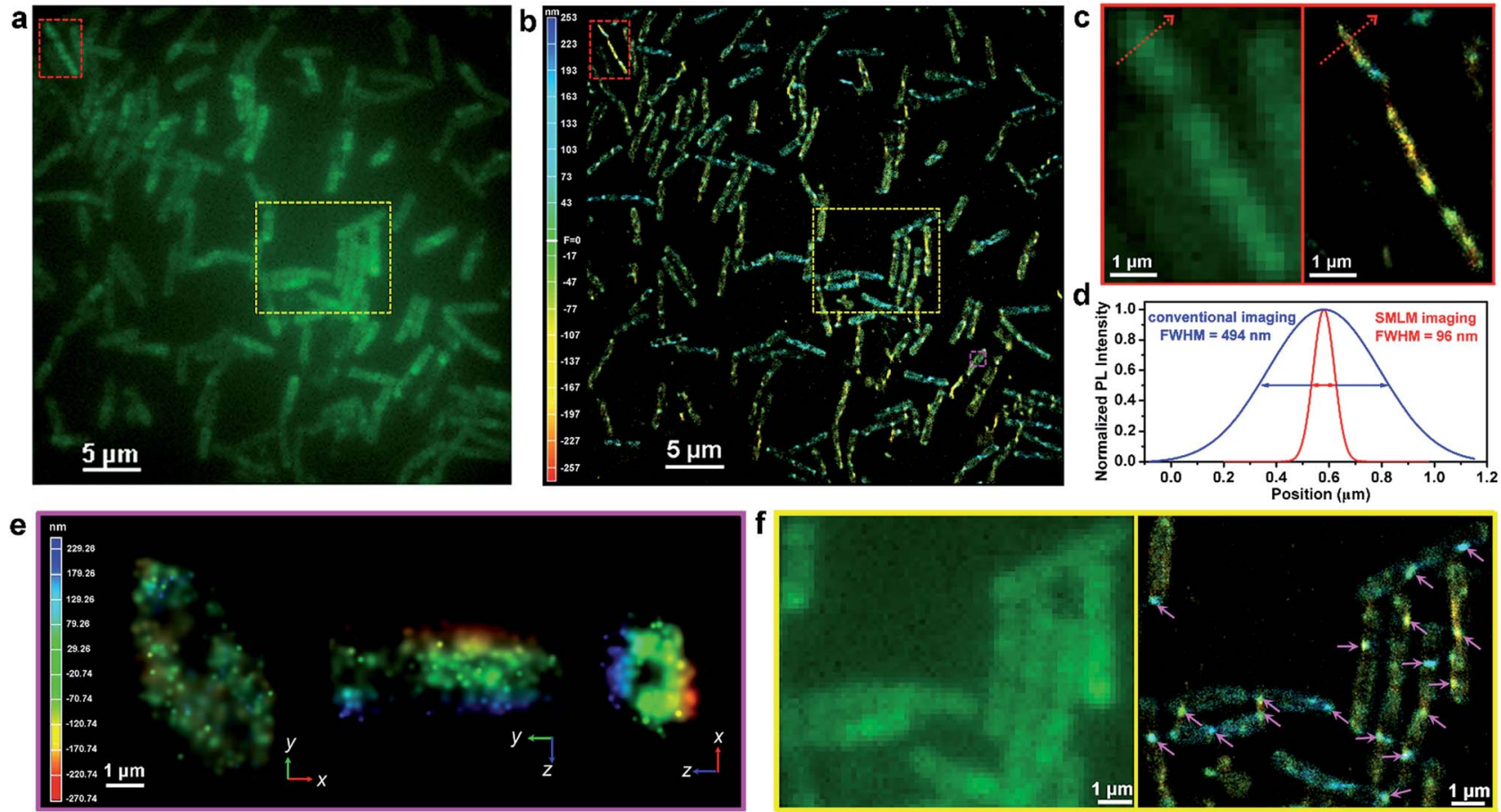

Fig. 5 (a) Conventional diffraction-limited fluorescence image and (b) 3D-SMLM image of live Bacillus subtilis cells stained with P18 in pH 4.5 buffer solution; the SMLM image contains ca. $1.24 \times 10^{6}$ localized positions, each plotted as a Gaussian with the full width at half-maximum (FHWM) in the $X$ and $Y$ direction. (c) Comparison of the imaging resolution of the three adjacent Bacillus subtilis cells in the enlarged region, as highlighted by red rectangles. (d) Gaussian fittings to the normalized PL intensities across a randomly selected bacterium (cross-sectional analysis along the red arrow). (e) $x y, y z$, and $x z$ views of the bacterial cell membranes shown in the pink square in (b), revealing the hollow cylindrical structure of the bacterial cell membrane. (f) Comparison of the imaging resolution in the enlarged region, as highlighted in yellow rectangles; the right SMLM image shows many fluid microdomains, as indicated by the pink arrows. 
In comparison to a conventional diffraction-limited fluorescence image (Fig. 4f), the 3D-SMLM image of Bacillus subtilis displayed a greatly improved spatial resolution (Fig. 4g). Besides, owing to the low intensity of the $405 \mathrm{~nm}$ excitation laser, and slow photobleaching rates of the $561 \mathrm{~nm}$ laser (as a result of fast ring-close reactions and a short open-form lifetime), P18 exhibited excellent photostability. The number of collected photons per 3000 frames ( $20 \mathrm{~ms}$ per frame) remained almost constant throughout our experiments of $>10 \mathrm{~min}$ (Fig. 4h), demonstrating the potential of P18 to perform long time-lapse nanoscopy imaging in future studies.

We continued to investigate the staining properties and labeling specificity of P18. As shown in Fig. 5a, b and Movie $1, \dagger$ P18 stained bacteria evenly with high density, and exhibited excellent photoblinking properties. Interestingly, the divisions of three Bacillus subtilis cells were clearly visible in a 3D-SMLM image (Fig. 5b and c), while they were barely resolved in a diffraction-limited fluorescence image (Fig. 5a and c). The fullwidth at half-maximum (FWHM) of a single Bacillus subtilis is $\sim 96 \mathrm{~nm}$ in the super-resolution images, $c f . \sim 494 \mathrm{~nm}$ in the conventional fluorescence images (Fig. 5d). Cross-sections of a single Bacillus subtilis highlighted in the pink box show a hollow structure from the $x z$ view, indicating that our probes localized on the cell membranes of bacteria (Fig. 5e). The flipped 3D-SMLM image of bacterial cell membranes is further shown in Movie 2. $\dagger$ We thus demonstrated that P18 enabled SMLM studies of the membranes of bacteria cells at nanometric resolution (without using additional targeting strategies). Moreover, we found that the membranes of most Bacillus subtilis bacteria did not show uniform fluorescence intensities in the 3D-SMLM image shown in Fig. 5b. The slight irregularity of the membrane staining likely indicates the presence of many fluid microdomains (as highlighted by pink arrows in the right image of Fig. 5f). These fluid microdomains had been well studied by several research groups. ${ }^{12}$ However, these fluid microdomains were hardly resolved in conventional diffractionlimited fluorescence images (in the left image of Fig. 5f). Besides, we also discovered that the majority of these fluid microdomains appeared in certain areas, especially around cell poles.

\section{Conclusions}

In summary, this study presents an effective molecular engineering approach for developing acid-resistant rhodamine spirolactams with a dedicated photoswitchable property and short open-form lifetime. These unique characteristics resulted from intramolecular hydrogen bonding between the 3-amino substituents in the carboxyphenyl ring and the lactam moiety. Moreover, we have extended the photoactivation wavelengths of rhodamine spirolactams into the visible region $(>400 \mathrm{~nm})$, by conjugating a 6-phenylethynyl naphthalimide moiety to the spirolactams. The visible photoactivation and dedicated photoswitchable properties of our dyes make them highly reliable fluorescent probes for SMLM, as demonstrated by the successful labeling and imaging of Bacillus subtilis at $\mathrm{pH} 4.5$ using 3DSMLM. We believe that our work sheds light on rational engineering of fluorescent properties of rhodamine dyes to facilitate the advancement of super-resolution imaging techniques.

\section{Experimental section}

\section{Single-crystal structures}

Single crystals of P4, P6, P7 and P8 that were suitable for X-ray structural analysis were obtained by slow evaporation from a chloroform $/ n$-hexane solution at room temperature. Single crystal X-ray diffraction data were collected on an Xcalibur, Atlas, Gemini ultra-diffractometer using the $\omega$-scan mode with a graphite-monochromator and Mo K $\alpha$ radiation. The crystal structures were solved with the Superflip ${ }^{13}$ structure solution program and refined with the SHELXL ${ }^{14}$ refinement package, as implemented in OLEX2. ${ }^{15}$ All $\mathrm{H}$ atoms were placed geometrically and treated with riding constraints, and displacement parameters were derived from the $\mathrm{C}$ atoms to which they were attached. All $\mathrm{CH}_{2}$ groups had $U_{\mathrm{iso}}(\mathrm{H})$ values fixed at 1.2 times the $U_{\text {eq }}$ value of the attached $\mathrm{C}$ atom. $\mathrm{CH}_{3}$ groups were idealized as freely rotating groups, with $U_{\text {iso }}(\mathrm{H})$ values fixed at 1.5 times the $U_{\text {eq }}$ value of the attached $\mathrm{C}$ atom. $\mathrm{H}$ atoms on $\mathrm{N}$ atoms were located via Fourier difference map inspection and positionally refined isotropically with thermal parameters based upon the $\mathrm{N}$ atom to which they are bonded $\left(U_{\text {iso }}(\mathrm{H})=1.2 U_{\text {eq }}(\mathrm{N})\right)$. These data can be obtained free of charge from the Cambridge Crystallographic Data Centre (CCDC reference numbers: 1582847, 1901370-1901372†).

\section{Measurement of single molecule optical properties}

P17 was immobilized in polymer films to investigate the effects of the excitation laser power density on (1) the photon output from dyes, (2) background noise, and (3) localization precision. Polymer films were prepared using 1\% (by mass) solution of polymethyl methacrylate (PMMA) in toluene. The polymer films were doped with P17 (10 nM) and then spin-cast onto argonplasma-etched coverslips. After that, the coverslips were placed in the sample holder of an SMLM instrument for imaging. The intensity of the $405 \mathrm{~nm}$ photoactivation laser was adjusted to activate only a few molecules in each frame. The fluorescence images of the polymer film were acquired using an electron-multiplying charge-coupled device (EMCCD) camera at room temperature.

\section{Sample preparation and data analysis for single-molecule fluorescence imaging experiment}

Coverslips were cleaned by sonication for $10 \mathrm{~min}$ in each of the following: Milli-Q water, absolute ethyl alcohol, and acetone. The above coverslips were further boiled in a solution mixture of concentrated sulfuric acid and hydrogen peroxide (v/v, $7 / 3$ ) for $1 \mathrm{~h}$ and then washed with Milli-Q water and dried using nitrogen. $20 \mu \mathrm{L}$ of very dilute P18 (10 nM) in DMSO solution was dripped on the dry coverslips. Then, P18 with the $N$-hydroxysuccinimide ester adsorbed and reacted with the hydroxyl groups on the surface of the cleaned coverslip at a low density such that individual dye molecules could be clearly resolved. 
Then the surface of the cleaned coverslip was rinsed with PBS ( $\mathrm{pH}$ 7.4) to remove unbound $\mathbf{P 1 8}$ before imaging. Finally, the coverslip was placed on the sample holder of the SMLM instrument and illuminated with $561 \mathrm{~nm}$ laser light $(0.36 \mathrm{~kW}$ $\mathrm{cm}^{-2}$ ). 5000-10 000 frames were recorded at a frame rate of 20 ms per frame. Single-molecule fluorescence time trace was calculated for a single molecule by analyzing the total integrated signal in a $2 \times 2$ pixel region centered on the single molecule during each frame of the movie. The number of photons was calculated as the total intensity count minus the background intensity count, which was converted to photon number using the calibrated parameter for electron multiplication and analogto-digital conversion gain settings used during acquisition.

\section{Cell culture and CLSM imaging}

MCF-7 cells were grown on DMEM (Gibco) supplemented with $10 \%$ FBS (Gibco). In CLSM imaging, the MCF-7 cells were incubated with $1 \mathrm{~mL}$ of DMEM containing $10 \mu \mathrm{M}$ P11 (experimental group) or P12 (control group) and the images were collected at 0, 30, 60 and 120 min by using an ANDORTM live cell imaging system. Then, the adherent cells were further incubated with $1 \mathrm{~mL}$ of fresh DMEM containing LysoTracker Green DND-26 (LTG) $(0.1 \mu \mathrm{M})$ for another $30 \mathrm{~min}$. The adherent cells were washed 3 times with PBS ( $\mathrm{pH} 7.4)$ and utilized for CLSM imaging. In the experimental group, the CLSM images of MCF-7 cells were taken under 0-5 min continuous UV (375 nm) irradiation at $37^{\circ} \mathrm{C}$.

\section{Live bacteria sample preparation and labeling}

A single colony of Bacillus subtilis was inoculated in $5 \mathrm{~mL}$ of Luria-Bertani (LB) medium with shaking at $37{ }^{\circ} \mathrm{C}$. Subsequently, $1 \mathrm{~mL}$ of the Bacillus subtilis suspension was inoculated in $10 \mathrm{~mL}$ of fresh LB media, which was incubated for $5 \mathrm{~h}$ with shaking $(250 \mathrm{rpm})$ at $37{ }^{\circ} \mathrm{C}$ to achieve mid-log phase growth. After the Bacillus subtilis had grown to the mid-log phase, $1 \mathrm{~mL}$ of the suspension was washed at least 3 times by centrifuging for 3 minutes at 10000 RPM and resuspending the pellet in $1 \mathrm{~mL}$ of clean PBS ( $\mathrm{pH}$ 4.5). To label the surface of Bacillus subtilis, $50 \mu \mathrm{L}$ of $\mathbf{P 1 7}$ in DMSO solution $(\sim 10 \mathrm{nM})$ was added slowly to the suspension of the Bacillus subtilis cells and left to incubate for 30 minutes. The unreacted $\mathbf{P 1 7}$ was rinsed 6 times with PBS ( $\mathrm{pH} 4.5$ ) before imaging. Then, the cleaned Bacillus subtilis cells were resuspended in a small amount of $\mathrm{PBS}(\mathrm{pH}$ 4.5) to produce a concentrated cell suspension. Finally, $2 \mu \mathrm{L}$ of this cell suspension was deposited onto an agarose pad $(1.5 \%$ (by mass) low melting point agarose in PBS) and mounted onto an Ar plasma etched glass slide (size: $24 \times 50 \mathrm{~mm}$, thickness: $0.13-0.16 \mathrm{~mm}$ ) and immediately used for the SMLM imaging.

\section{D-SMLM imaging and data processing}

The super-resolution imaging was carried out using a Nikon NSTORM 5.0 super-resolution microscope system with a motorized inverted microscope ECLIPSE Ti2-E, a 100×/NA 1.49 oil immersion TIRF objective lens (CFI HP) and an ORCA-Flash 4.0 sCMOS camera (Hamamatsu Photonics K.K.). During superresolution imaging, a $405 \mathrm{~nm}$ laser $\left(1.8 \mathrm{~W} \mathrm{~cm}^{-2}\right)$ was employed to photoactivate rhodamine spirolactams from ringclosed isomers to ring-opened isomers. At the same time, a $561 \mathrm{~nm}$ laser $\left(1.2 \mathrm{~kW} \mathrm{~cm}{ }^{-2}\right)$ was used as the excitation source to produce sparse emissions from ring-opened rhodamines. Repeating this cycle many times (i.e., to collect $\sim 30000$ images with the ${ }_{\mathrm{s}} \mathrm{CMOS}$ camera with an integration time of $20 \mathrm{~ms}$ per frame) allowed us to reconstruct a super-resolution image. Astigmatism was used in the 3D-imaging experiments. The software NIS-Elements Ar and N-STORM Analysis were used to analyze the collected images and computationally reconstruct the 3D-SMLM image.

\section{Conflicts of interest}

The authors declare no conflict of interest.

\section{Acknowledgements}

This work was supported by the National Natural Science Foundation of China (21708039, 21502189, 21878286), CPSF (2017M611278, 2018T110234), and DICP (DMTO201603, TMSR201601, 2015YB07). X. L. and W. C. are indebted to the financial support from SUTD (T1SRCI17126, IDD21700101, IDG31800104). J. Y. acknowledges the support from the National Research Foundation of Korea (NRF) (No. 2012R1A3A2048814).

\section{Notes and references}

1 (a) S. W. Hell, Science, 2007, 316, 1153; (b) A. von Diezman, Y. Shechtman and W. E. Moerner, Chem. Rev., 2017, 117, 7244; (c) E. Betzig, Angew. Chem., Int. Ed., 2015, 54, 8034.

2 G. Patterson, M. Davidson, S. Manley and J. LippincottSchwartz, Annu. Rev. Phys. Chem., 2010, 61, 345.

3 (a) M. Heilemann, S. van de Linde, M. Schuttpelz, R. Kasper, B. Seefeldt, A. Mukherjee, P. Tinnefeld and M. Sauer, Angew. Chem., Int. Ed., 2008, 47, 6172; (b) J. B. Grimm, T. Klein, B. G. Kopek, G. Shtengel, H. F. Hess, M. Sauer and L. D. Lavis, Angew. Chem., Int. Ed., 2016, 55, 1723.

4 (a) O. Nevskyi, D. Sysoiev, A. Oppermann, T. Huhn and D. Wöll, Angew. Chem., Int. Ed., 2016, 55, 12698; (b) B. Roubinet, M. L. Bossi, P. Alt, M. Leutenegger, H. Shojaei, S. Schnorrenberg, S. Nizamov, M. Irie, V. N. Belov and S. W. Hell, Angew. Chem., Int. Ed., 2016, 55, 15429; (c) B. Roubinet, M. Weber, H. Shojaei, M. Bates, M. L. Bossi, V. N. Belov, M. Irie and S. W. Hell, J. Am. Chem. Soc., 2017, 139, 6611.

5 (a) H. N. Kim, M. H. Lee, H. J. Kim, J. S. Kim and J. Yoon, Chem. Soc. Rev., 2008, 37, 1465; (b) X. Chen, T. Pradhan, F. Wang, J. S. Kim and J. Yoon, Chem. Rev., 2012, 112, 1910. 6 (a) K. H. Knauer and R. Gleiter, Angew. Chem., Int. Ed., 1977, 16, 113; (b) H. Willwohl, J. Wolfrum and R. Gleiter, Laser Chem., 1989, 10, 63; (c) H. Montenegro, M. D. Paolo, D. Capdevila, P. F. Aramendia and M. L. Bossi, Photochem. Photobiol. Sci., 2012, 11, 1081; (d) K. Li, Y. Xiang, X. Wang, J. Li, R. Hu, A. Tong and B. Z. Tang, J. Am. Chem. Soc., 2014, 136, 1643. 
7 (a) J. Folling, V. Belov, R. Kunetsky, R. Medda, A. Schonle, A. Egner, C. Eggeling, M. Bossi and S. W. Hell, Angew. Chem., Int. Ed., 2007, 46, 6266; (b) V. N. Belov, M. L. Bossi, J. Fölling, V. P. Boyarskiy and S. W. Hell, Chem.-Eur. J., 2009, 15, 10762; (c) M. K. Lee, P. Rai, J. Williams, R. J. Twieg and W. E. Moerner, J. Am. Chem. Soc., 2014, 136, 14003; (d) B. Roubinet, M. Bischoff, S. Nizamov, S. Yan, C. Geisler, S. Stoldt, G. Y. Mitronova, V. N. Belov, M. L. Bossi and S. W. Hell, J. Org. Chem., 2018, 83, 6466; (e) M. Bossi, J. Folling, V. N. Belov, V. P. Boyarskiy, R. Medda, A. Egner, C. Eggeling, A. Schonle and S. W. Hell, Nano Lett., 2008, 8, 2463; (f) V. N. Belov, C. A. Wurm, V. P. Boyarskiy, S. Jakobs and S. W. Hell, Angew. Chem., Int. Ed., 2010, 49, 3520; (g) J. Folling, V. Belov, D. Riedel, A. Schonle, A. Egner, C. Eggeling, M. Bossi and S. W. Hell, ChemPhysChem, 2008, 9, 321; (h) H. Aoki, K. Mori and S. Ito, Soft Matter, 2012, 8, 4390; (i) E. A. Halabi, D. Pinotsi and P. Rivera-Fuentes, Nat. Commun., 2019, 10, 1232; (j) E. A. Halabi, Z. Thiel, N. Trapp, D. Pinotsi and P. RiveraFuentes, J. Am. Chem. Soc., 2017, 139, 13200; $(k)$ E. A. Halabi, S. Püntener and P. Rivera-Fuentes, Helv. Chim. Acta, 2018, 101, e1800165.

8 V. N. Belov and M. L. Bossi, Isr. J. Chem., 2013, 53, 1.

9 (a) R. A. Gottlieb, H. A. Giesing, J. Y. Zhu, R. L. Engler and B. M. Babior, Proc. Natl. Acad. Sci. U. S. A., 1995, 92, 5965; (b) R. A. Gottlieb, J. Nordberg, E. Skowronski and B. M. Babior, Proc. Natl. Acad. Sci. U. S. A., 1996, 93, 654; (c)
M. A. Barry, J. E. Reynolds and A. Eastman, Cancer Res., 1993, 53, 2349.

10 (a) S. N. Uno, M. Kamiya, T. Yoshihara, K. Sugawara, K. Okabe, M. C. Tarhan, H. Fujita, T. Funatsu, Y. Okada, S. Tobita and Y. Urano, Nat. Chem., 2014, 6, 681; (b) H. Takakura, Y. Zhang, R. S. Erdmann, A. D. Thompson, Y. Lin, B. McNellis, F. Rivera-Molina, S. N. Uno, M. Kamiya, Y. Urano, J. E. Rothman, J. Bewersdorf, A. Schepartz and D. Toomre, Nat. Biotechnol., 2017, 35, 773. 11 G. Xi, L. Sheng, I. Zhang, J. Du, T. Zhang, Q. Chen, G. Li, Y. Zhang, Y. Song, J. Li, Y. Zhang and S. X. Zhang, ACS Appl. Mater. Interfaces, 2017, 9, 38032.

12 (a) K. Matsumoto, J. Kusaka, A. Nishibori and H. Hara, Mol. Microbiol., 2006, 61, 1110; (b) A. S. Johnson, S. van Horck and P. J. Lewis, Microbiology, 2004, 150, 2815; (c) F. Kawai, M. Shoda, R. Harashima, Y. Sadaie, H. Hara and K. Matsumoto, J. Bacteriol., 2004, 186, 1475; (d) I. Fishov and C. L. Woldringh, Mol. Microbiol., 1999, 32, 1166; (e) A. Nishibori, J. Kusaka, H. Hara, M. Umeda and K. Matsumoto, J. Bacteriol., 2005, 187, 2163.

13 O. V. Dolomanov, L. J. Bourhis, R. J. Gildea, J. A. K. Howard and H. Puschmann, J. Appl. Crystallogr., 2009, 42, 339.

14 L. Palatinus and G. Chapuis, J. Appl. Crystallogr., 2007, 40, 786.

15 G. M. Sheldrick, Acta Crystallogr., Sect. A: Found. Crystallogr., 2008, 64, 112. 